\title{
How to Design a Highly Organized Cell: An Unexpectedly High Number of Widely Diversified SNARE Proteins Positioned at Strategic Sites in the Ciliate, Paramecium tetraurelia
}

\section{Introduction}

There are only scattered data available on molecular aspects of vesicle trafficking in protozoa, notably in ciliates. In this context, proteins of paramount interest are the so-called SNARE proteins (soluble NSF attachment protein receptor; $\mathrm{NSF}=\mathrm{N}$-ethylmaleimide sensitive factor). They are positioned on opposite membranes; together with some other proteins they serve docking, e.g., of a vesicle to a target membrane (v-/t-SNAREs), and finally membrane fusion (Jackson and Chapman 2006; Jahn and Scheller 2006; Jahn et al. 2003; Malsam et al. 2008; Parlati et al. 2002; Söllner et al. 1993). In the cell, SNAREs contribute to the specificity of such interactions (Bethani et al. 2007; Parlati et al. 2002; Paumet et al. 2004), together with monomeric GTP-binding proteins (G-proteins, small GTPases [Grosshans et al. 2006; Rothman 1994]), the vesicular $\mathrm{H}^{+}$-ATPase (V-ATPase [Hurtado-Lorenzo et al. 2006; Pfeffer 2007]), F-actin (Soldati and Schliwa 2006) and a variety of additional proteins (Wojcik and Brose 2007). The assembly and function of SNAREs during vesicle docking and membrane fusion is outlined in Figure 1.

We now have systematically identified SNAREs in Paramecium tetraurelia and analyzed their distribution and basic aspects of their function (Kissmehl et al. 2007; Schilde et al. 2006, 2008, 2010), together with the SNARE-specific chaperone, NSF (Froissard et al. 2002; Kissmehl et al. 2002), in these cells. This is summarized here with the aim to set a baseline for future research on this fundamental aspect of vesicle trafficking. No comparably comprehensive analyses are available from any other free-living protozoan, but as far as possible such data are included, as are some data from algae. It is challenging to see how these data

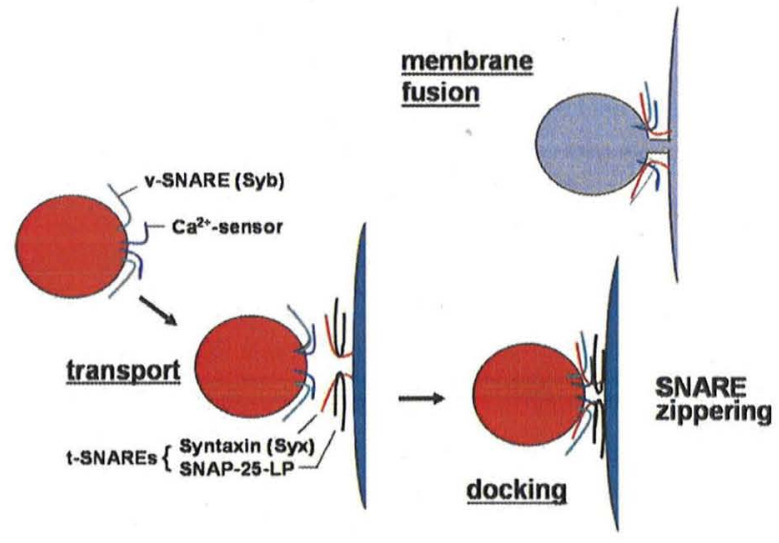

Figure 1. Details on essential steps and molecular components in vesicle-target membrane interaction. Steps following intracellular transport include tethering, docking (accompanied by SNARE zippering) and fusion. Essential components depicted on vesicles are v-SNAREs (type synaptobrevin, Syb) and a $\mathrm{Ca}^{2+}$-sensor which, however, does not participate in all intracellular membrane interactions and whose identity (e.g., for trichocyst exocytosis) is not known in ciliates. On the target side, two (or three) types of t-SNAREs may occur (see text), i.e., type syntaxin (Syx) and SNAP-25-like protein (SNAP-25-LP). By backfolding, the latter contributes two SNARE domains to a quarternary trans-SNARE complex, whereas all other SNAREs contribute only one. SNAP-25 and similar SNARE proteins possess no carboxy-terminal trans-membrane domain for anchoring, in contrast to other SNAREs. Eventually the function of SNAP-25-LP can be exerted by to separate SNARE proteins. For membrane fusion to occur, each of the two membranes involved must contain at least one SNARE with a trans-membrane domain. This rough outline also matches the situation in Paramecium. 
compare with higher eukaryotes, considering the complex diversification vesicles and their proteins have achieved during evolution.

\section{Paramecium as a Model System - From Past to Present}

Over a long time, Paramecium cells have served as a model system for several important aspects of vesicle trafficking. Figure 2 outlines some of the most essential trafficking pathways in Paramecium. This includes the classical pathways of exo- and endocytosis, the phagocytotic pathway, and the osmoregulatory system. Paramecium was important for the analysis of principal steps of the formation and processing of phagosomes/phagolysosomes ("food vacuoles", "digestive vacuoles"). This includes acidification of phagosomes, after pinching off, by fusing with "acidosomes" and fusion with lysosomes to form a phagolysosome, followed by recycling of food vacuole membrane components via "discoidal" and other vesicles (Allen and Fok 2000; Fok and Allen 1990). Another pathway deals with exocytosis and endocytosis. This transport route includes processing of secretory materials in the dense core-secretory vesicles, the "trichocysts", which not only mediates highest packing density of crystalline contents but also competence for docking at the cell membrane (Gautier et al. 1994). Stimulation initiates $\mathrm{Ca}^{2+}$-dependent exocytotic membrane fusion (Plattner and Klauke 2001), contents discharge and membrane retrieval by exocytosiscoupled endocytosis (Plattner et al. 1993; Vayssié et al. 2000). For some of these aspects, e.g., exocytosis and exocytosis-coupled endocytosis, Paramecium cells were an important model system because they could be analyzed on a finely tuned sub-second time scale due to an unsurpassed degree of synchrony (Plattner and Hentschel 2006), much more than available with any other dense core-vesicle system (Kasai 1999). A third main trafficking route is represented by the contractile vacuole complex/osmoregulatory system (Fig. 2).

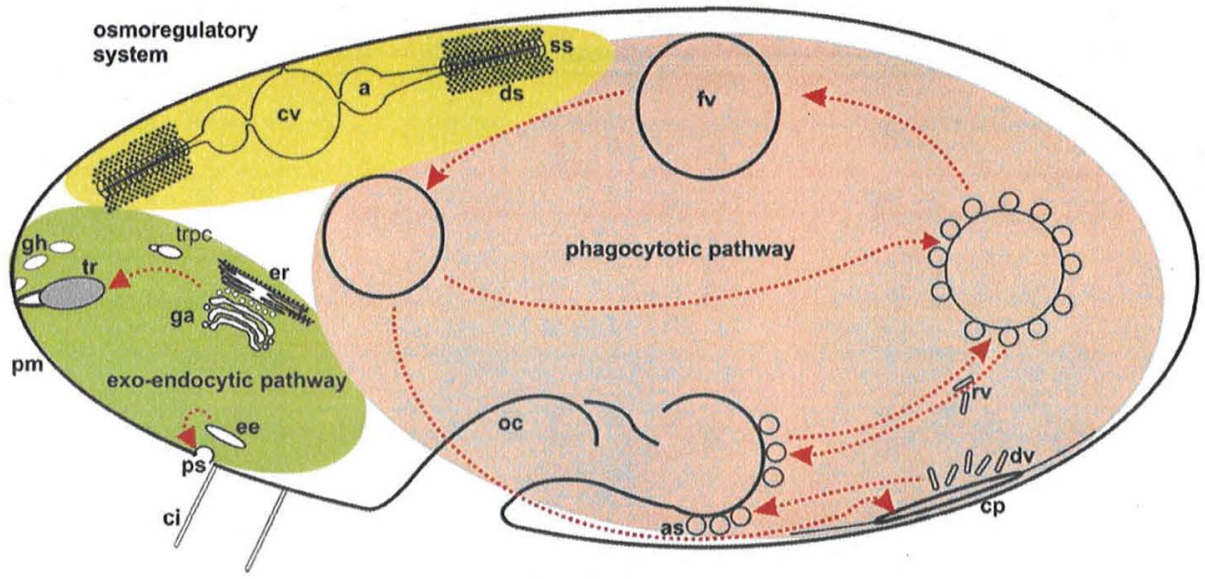

Figure 2. Main vesicle trafficking pathways in a Paramecium cell highlighted in green (exo-endocytotic pathways), red (phago[lyso]somal system) and yellow (contractile vacuole complex). Note that the scheme cannot take into consideration that a Paramecium cell usually contains two contractile vacuole complexes, many Golgi fields, and even many more trichocyst docking/stimulated exocytosis sites, as well as endocytosis sites and endosomes, together with a multitude of cilia. Also, the Golgi apparatus produces vesicles other than trichocyst precursor vesicles (see text). Widely different vesicle types, in addition to acidosomes, are also associated with the oral cavity (not depicted), as commented in the text. Abbreviations: $\mathrm{a}=$ ampullae, as=acidosomes (late endosomes), $\mathrm{ci}=\mathrm{cilia}, \mathrm{cp}=\mathrm{cytoproct}$ (exocytosis site for spent food vacuoles), $\mathrm{cv}=$ contractile vacuole, $\mathrm{ds}=$ decorated spongiome, $\mathrm{dv}=$ discoidal vesicles (recycling from cytoproct), ee=early endosomes ("terminal cisternae"), er=Endoplasmic Reticulum, fv=food vacuole (originating as a phagosome, maturing to a phagolysosome), ga=Golgi apparatus, gh="ghosts" from trichocysts released by exocytosis, oc=oral cavity, pm=plasma membrane, ps="parasomal sacs" (coated pits), rv=recycling vesicles (from maturing food vacuole), $s s=s m o o t h$ spongiome, svcy=small vesicles undergoing cyclosis (see Figures 5 to 7), tr=trichocysts, trpc=trichocyst precursor organelles. 
In many cases work with Paramecium has been paradigmatic over a considerable time, but over the years lack of a genomic database has hampered any further progress with this system. At the same time work with "higher" eukaryotes, based on new technologies, including genetic manipulation (gene silencing or knockout, sitedirected mutagenesis, overexpression as green fluorescent protein-[GFP-]fusion proteins) and novel electrophysiological methods (patch-clamp analysis and amperometry), etc. had progressed to a standard not yet available for ciliate biology.

Only recently could we catch up with this development, thus enabling us not only to achieve a methodological standard almost equivalent to that of mammalian cells, but also to exploit some of the special features that had made Paramecium a model system for a long time. Consider that these cells have a highly regular design, with clear-cut pathways for several vesicle populations (Allen and Fok 2000; Fok and Allen 1990; Plattner 2002), an unexpectedly dynamic contractile vacuole system serving the regulation of osmotic and ionic homeostasis (Allen and Naitoh 2002), a considerable number of secretory mutants (Beisson et al. 1976; Vayssié et al. 2000) and last not least, the fastest dense core-vesicle release system known (Plattner and Hentschel 2006; Plattner and Kissmehl 2003; Plattner et al. 1993).

\section{A Brief Sketch of SNARE Protein Structure and Function}

SNARE proteins usually are anchored at their carboxy-terminal side by a single transmembrane domain. This is followed by a SNARE domain, between $\sim 60$ and 70 amino acids long. The $\alpha$-helical SNARE domain contains heptad repeats arranged around a so-called "zero-layer" which usually is represented by an arginine (Arg, R) and a glutamine (Gln, Q) residue (Fasshauer et al. 1998), respectively, in the SNAREs of opposite membranes. For a general summary, see Jahn et al.

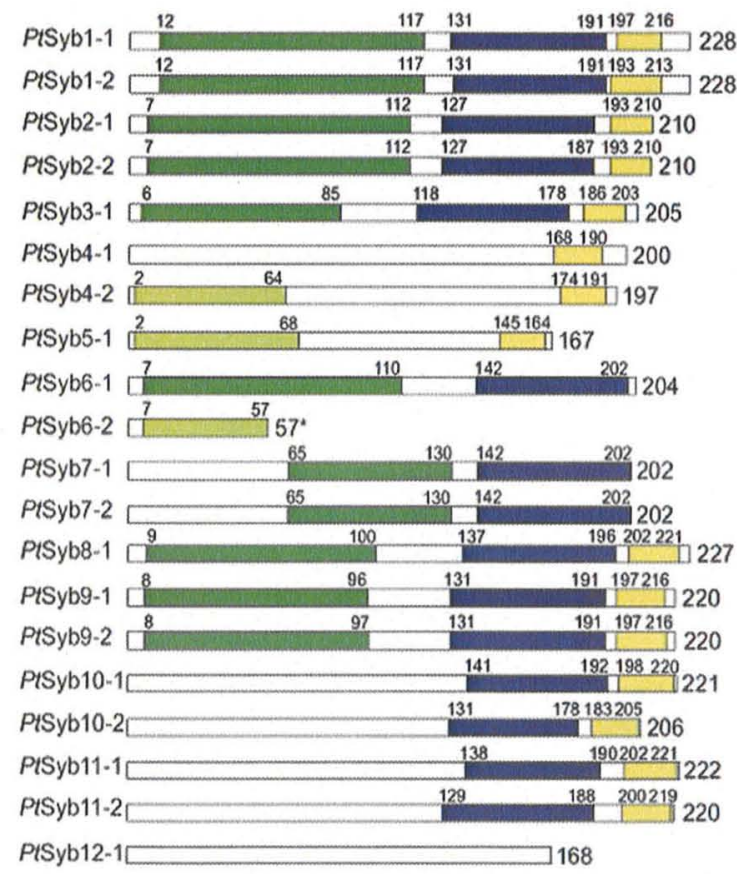

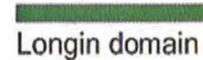

Longin domain with low score

Synaptobrevin

coiled-coil homology (SNARE domain)

predicted

transmembrane

domain

Figure 3. Example of the varying organization of synaptobrevin-like SNAREs in Paramecium. Note the varying occurrence of domains, such as a trans-membrane domain, a SNARE-domain and a longin-domain. Also consider the variable position and length of domains, although their sequence is stereotypically arranged. Molecular variations within the SNARE domain of PtSyb paralogs and ohnologs are exemplified in Figure 4. Also note that a transmembrane domain may be missing; such truncation, though exceptional, also occurs in other organisms. Similar molecular variations as in PtSyb-type SNAREs also occur in syntaxin-like SNAREs of Paramecium.

From Schilde et al. (2010) with permission. 


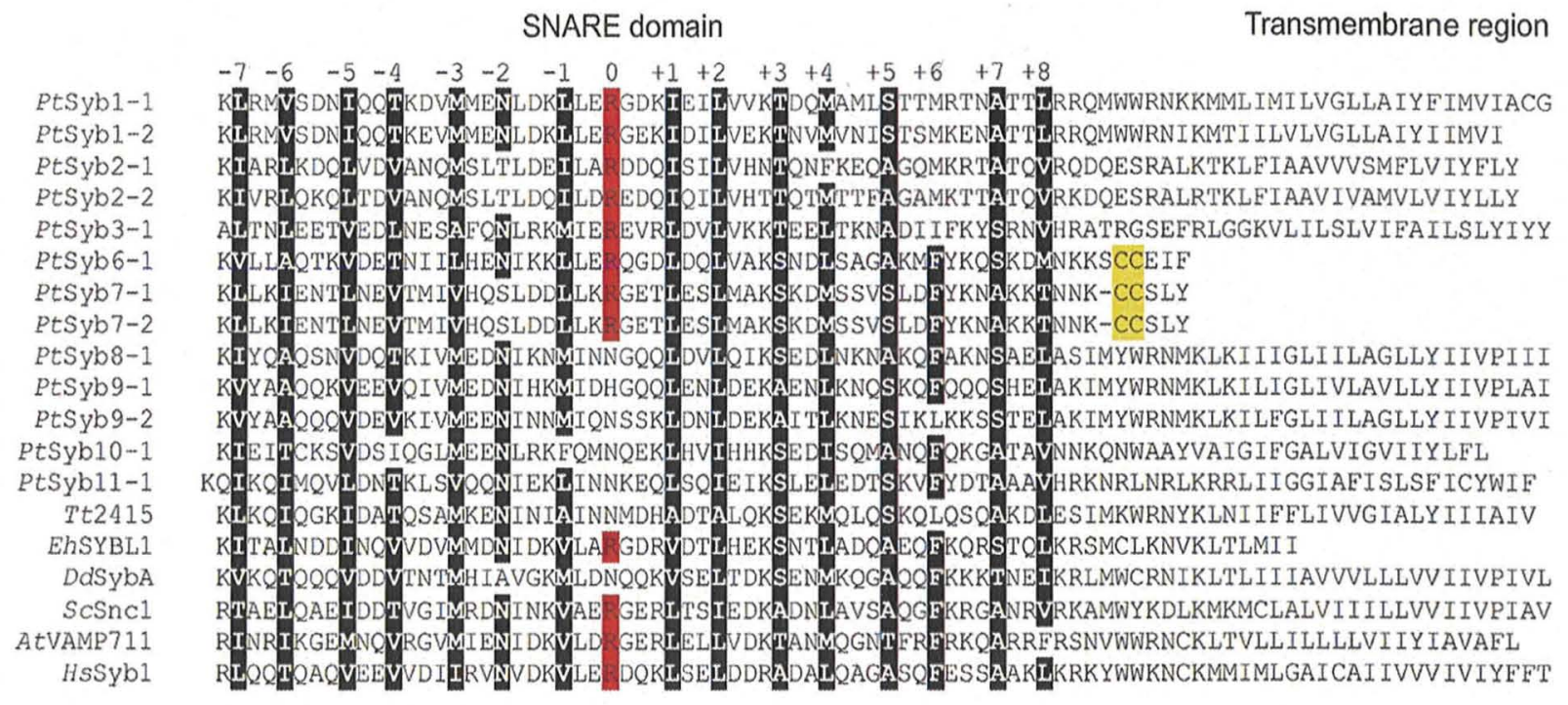

Figure 4. Details of SNARE domains of PtSyb paralogs and ohnologs. Centered around the zero-layer are heptad repeats (black background), as explained in the text. While $\mathrm{R}$ is the orthodox residue (red) in the zerolayer of synaptobrevins, there are exceptions to this rule, in Paramecium as well as in other organisms up to mammals. Moreover, some heptat repeats display interruptions (where black background is absent). Also note that carboxy-terminal double cystein residues (yellow) would be appropriate for lipophilic derivatization, although this aspect has not yet been analyzed in depth in ciliates. For species abbreviations, see Figure 8. From Schilde et al. (2010) with permission.

(2003), for examples of the situation in Paramecium, see Figures 3 and 4 . The association of SNAREs with the opposite membranes led to a dual nomenclature, $v$-/t-SNAREs, which generally correspond to R-/Q-SNAREs, although there occur frequent deviations from these designations.

Typical examples of SNAREs are synaptobrevin (Syb) for v-/R-SNAREs and syntaxin (Syx) for t-/QSNAREs, respectively. The situation with t-/QSNAREs is more complex than with v-/R-SNAREs. There are several types ( $\mathrm{Qa}, \mathrm{Qb}$ and $\mathrm{Qc}$, or $\mathrm{Qb} / \mathrm{c}$, as explained below) which can contribute to a functional SNARE complex. In vivo, this complex is made of three or four SNARE molecules, each with one $\alpha$-helical SNARE domain (or two, when contained in a hairpin-shaped Qb/c SNARE). Thus a quaternary trans-SNARE complex is formed during SNARE pairing which finally can lead to membrane fusion. In sum, t-/Q-SNAREs $(\mathrm{Q} a+\mathrm{Qb}+\mathrm{Qc}$, or $\mathrm{Qa}+\mathrm{Qb} / \mathrm{c})$ on one of the membranes always associate with one $\mathrm{v}$-/RSNARE on the other membrane (Jahn et al. 2003), to a quaternary SNARE complex.

Let us consider this in more detail from another point of view. When SNAREs from opposite membranes interact during docking, SNAREs pair to a complex by "SNARE zippering" from the amino-terminus to the carboxy-terminal anchor, thus reducing the distance between the two membranes to be fused (Lin and Scheller 1997; Melia et al. 2002; Pobbati et al. 2006; Sørensen et al. 2006). Accordingly, SNAREs were also subdivided, as mentioned, into vesicle and target-SNAREs, v- and t-SNAREs, respectively. The three or four SNAREs arrange themselves in a way that the zero-layers of their four SNARE domains are positioned side-by-side in one plane whereby they are stabilized by hydrogen bonds between the three glutamine residues $(\mathrm{Qa}+\mathrm{Qb}+\mathrm{Qb}$ or $\mathrm{Qa}+\mathrm{Qb} / \mathrm{c}$ ) and the guanidino group of the arginine (R) side chain (Fasshauer et al. 1998; Sutton et al. 1998). This is called the " $3 Q+1 R$-rule" of SNARE pairing. In summary, a quarternary SNARE complex (four helical bundles) is formed during docking and this prepares the fusion process. It also has to be noted that there are many exceptions to some of the characteristics, as is the case also with Paramecium SNAREs (Fig. 4).

Additional general aspects of SNARE structure and function, also of importance for ciliates, are the following. Some forms of synaptobrevins would better be named "longins" when they possess an amino-terminal "longin" domain of 
$\sim 100$ to 140 amino acids (Filippini et al. 2001; Rossi et al. 2004), as is the case in Paramecium (Fig. 3). As mentioned, a typical Q-SNARE is the Qa-SNARE syntaxin. Syntaxin possesses, in addition to the transmembrane and to the SNARE domains, a most distal syntaxin domain, also called the Habc-domain of $\sim 47$ to 71 amino acids, (Bock and Scheller 1996; Rizo and Südhof 2002). This domain serves the sequential attachment of $\alpha$-SNAP and NSF. The $\alpha$-SNAP molecule (unrelated to the Qb/c SNARE, SNAP-25) is an adaptor for the binding of NSF, the SNARE-specific chaperone (see below).

In vivo, SNAREs display a rather distinct topology, thus suggesting a role in specifying membrane interactions, in combination with other, eventually exchangeable molecular components. All these details are of particular importance for identifying SNAREs. What makes analyses difficult is the fact that there are exceptions to most of the rules in all organisms - as we have experienced also with Paramecium.

\section{Towards Identification of SNAREs in Paramecium}

An important step towards understanding the $P$. tetraurelia cell on a molecular basis has been the analysis of numerous mutants Nayssié et al. 2000), e.g., by complementation cloning (Haynes et al. 1996; Skouri and Cohen 1997). Several secretory mutants could thus be elucidated at the molecular level (Vayssié et al. 2000) although no SNAREs had been found in this important work. Analyses have then been extended to the generation of an indexed macronuclear genomic library (Keller and Cohen 2000), followed by gene cloning and annotation (Dessen et al. 2001). Finally the $P$. tetraurelia genome has been annotated (Aury et al. 2006; Zagulski et al. 2004) and a database (ParameciumDB) became available (Arnaiz et al. 2007): 〈http://paramecium. cgm.cnrs-gif.fr/ $>$.

On the way to this stage of methodological development, many genes and their transcripts have been analyzed in detail for an unequivocal identification and for functional implications. We summarize these data in Tables 1 and 2 where we also provide accession numbers. We include those from our manual annotations (controlled and supplemented by expression studies) and those from automatic annotations in the ParameciumDB (including some additional data). Along these lines, our laboratory has concentrated on the analysis of SNAREs (Kissmehl et al. 2007; Schilde et al. 2006, 2008, 2010) and the SNAREspecific chaperone, NSF (Froissard et al. 2002; Kissmehl et al. 2002) in P. tetraurelia.

In other unicellular organisms, particularly in free-living forms (except yeast), SNAREs have hardly been subjected to any comparable analysis with respect to molecular structure, expression, localization and function. For identification and assessment of functional implications, the Paramecium database has been BLAST-searched, concentrating on characteristic domain structures. This is recently supported by a SNARE database from the Max Planck Institute, Göttingen: 〈http://www.mpibpc.mpg.de/english/ service/bioinformatics/index.html > or 〈http:// bioinformatics.mpibpc.mpg.de/snare/index.jsp $\rangle$ using an algorithm trained in recognizing characteristic features of SNARE molecules. This has been used in combination with the $P$. tetraurelia macrogenomic database for manual annotations. Expression has been controlled on the basis of an expression library/cDNA analysis, followed by antibody production for Western blots, light and electron microscopic (EM) immuno-localization as well as by expression as GFP-fusion proteins (Hauser et al. 2000) and gene silencing. The latter has become possible by the development of an RNA interference (RNAi) methodology (Galvani and Sperling 2002; Meyer and Cohen 1999; Ruiz et al. 1998).

For paralogs originating from whole genome duplication (WGD) Wolfe (2004) has coined the term "ohnologs", thus honoring concomitant analyses by the geneticist Ohno (1970). In yeast, for instance, such genes - if not deleted during evolution - may become functionally diversified to a different extent (Langkjaer et al. 2003). In general, in Paramecium, such ohnologs are frequently retained. Ohnologs originating from a most recent WGD may serve gene amplification, rather than functional/topological diversification (Aury et al. 2006). We will have to consider this aspect below for SNARE ohnologs in Paramecium, where in many cases pairs, in other cases, only singletons remain.

In $P$. tetraurelia SNARE pairs derived from the last WGD (ohnologs) are frequently very similar to each other and they appear to have largely identical localization and function. Along these lines, close similarity ( $\geq 85 \%$ on a nucleotide basis) restrains one from selective functional analysis by gene silencing or, vice versa, this high similarity frequently offers the possibility to silence two ohnologs at a time. This has been found 
Table 1. Synaptobrevin-like SNAREs in P. tetraurelia. Data collected from Schilde et al. $(2006,2008,2010)$ and Kissmehl et al. (2007) who also provide accession numbers from manual annotations. See also automatic annotations in the ParameciumDB which also includes PtSec22.

\begin{tabular}{|c|c|c|c|c|c|c|c|}
\hline \multirow[t]{2}{*}{ Designation } & \multicolumn{2}{|c|}{ annotation/accession numbers } & \multirow{2}{*}{$\begin{array}{l}\text { Trans- } \\
\text { membrane } \\
\text { domain }\end{array}$} & \multirow{2}{*}{$\begin{array}{l}\text { SNARE } \\
\text { domain }\end{array}$} & \multirow{2}{*}{$\begin{array}{l}\text { zero } \\
\text { layer }\end{array}$} & \multirow{2}{*}{$\begin{array}{l}\text { longin } \\
\text { domain }\end{array}$} & \multirow{2}{*}{$\begin{array}{l}\text { established } \\
\text { SNARE character?/ } \\
\text { remarks }\end{array}$} \\
\hline & manual & automatic & & & & & \\
\hline PtSyb1-1 & AJ566298 & GSPATG00008957001. & present & present & $R$ & present & yes \\
\hline PtSyb1-2 & CR855907 & GSPATG00025290001 & present & present & $R$ & present & yes \\
\hline PtSyb2-1 & AJ566299 & GSPATG00023002001 & present & present & $\mathrm{R}$ & present & yes \\
\hline PtSyb2-2 & AJ566300 & GSPATG00021098001 & present & present & $R$ & present & yes \\
\hline PtSyb3-1 & AJ566301 & GSPATG00030667001 & present & present & $R$ & present & yes \\
\hline PtSyb4-1 & CAK60380 & GSPATGO0005129001 & present & absent & - & present & yes/homology to PtSyb3 \\
\hline PtSyb4-2 & CAK94759 & GSPATG00027083001 & present & absent & - & present & yes/homology to PtSyb3 \\
\hline PtSyb5-1 & CAK73681 & GSPATG00009953001 & present & absent & - & present & yes/homology to PtSyb3 \\
\hline PtSyb6-1 & CR855902 & GSPATG00011479001 & absent & present & $R$ & present & yes \\
\hline (PtSyb6-2) & CR855978 & GSPATG00011185001 & & & & fragmer & no functional SNARE \\
\hline PtSyb7-1 & CR855901 & GSPATG00038192001 & absent & present & $R$ & present & yes \\
\hline PtSyb7-2 & CR855900 & GSPATG00021245001 & absent & present & $R$ & present & yes \\
\hline PtSyb8-1 & CAK63760 & GSPATG00033613001 & present & present & $N$ & present & yes \\
\hline PtSyb9-1 & CAK63650 & GSPATG00033503001 & present & present & $\mathrm{H}$ & present & yes \\
\hline PtSyb9-2 & CAK73996 & GSPATG00010230001 & present & present & $N$ & present & yes \\
\hline PtSyb10-1 & CAK90226 & GSPATG00023401001 & present & present & $N$ & absent & yes \\
\hline PtSyb10-2 & CAK88554 & GSPATG00021722001 & present & present & $\mathrm{N}$ & absent & yes \\
\hline PtSyb11-1 & CAK75272 & GSPATG00001475001 & present & present & $N$ & absent & yes \\
\hline PtSyb11-2 & CAK85181 & GSPATG00002581001 & present & present & $N$ & absent & yes \\
\hline$(P+S y b 12-1)$ & CAK87252 & GSPATG00020905001 & absent & absent & - & absent & $\begin{array}{l}\text { no/only overall similarity to } \\
\text { SNARE sequences }\end{array}$ \\
\hline PtSec22 & & GSPATO0009443001 & present & present & $\mathrm{R}$ & present & yes \\
\hline
\end{tabular}


Table 2. Syntaxin-like SNAREs in P. tetraurelia. Data collected from Kissmehl et al. (2007) and Schilde et al. (2008; for PtSNAP-25-LP) who also provide accession numbers from manual annotations (GenBank, Genoscope); see also automatic annotations in the ParameciumDB which also includes PtSNAP-25-LP.

\begin{tabular}{|c|c|c|c|c|c|c|}
\hline \multirow[t]{2}{*}{ Designation } & \multicolumn{2}{|c|}{ annotation/accession numbers } & \multirow{2}{*}{$\begin{array}{l}\text { Trans-membrane } \\
\text { domain }\end{array}$} & \multirow{2}{*}{$\begin{array}{l}\text { SNARE } \\
\text { domain }\end{array}$} & \multirow[t]{2}{*}{ zero layer } & \multirow{2}{*}{$\begin{array}{l}\text { established } \\
\text { SNARE character?/ } \\
\text { remark }\end{array}$} \\
\hline & manual & automatic & & & & \\
\hline $\begin{array}{l}\text { PtSyx1-1 } \\
\text { PtSyx1-2 } \\
\text { PtSyx2-1 } \\
\text { PtSyx2-2 } \\
\text { PtSyx3-1 } \\
\text { PtSyx3-2 } \\
\text { PtSyx4-1 } \\
\text { PtSyx4-2 } \\
\text { PtSyx5-1 } \\
\text { PtSyx5-2 } \\
\text { PtSyx6-1 } \\
\text { PtSyx7-1 } \\
\text { PtSyx7-2 } \\
\text { PtSyx8-1 } \\
\text { PtSyx8-2 } \\
\text { PtSyx9-1 } \\
\text { PtSyx9-2 } \\
\text { PtSyx10-1 } \\
\text { PtSyx10-2 } \\
\text { PtSyx11-1 } \\
\text { PtSyx12-1 } \\
\text { (PtSyx13-1) } \\
\text { PtSyx14-1 } \\
\text { PtSyx14-2 } \\
\text { PtSyx15-1 } \\
\text { PtSNAP-25-LP }\end{array}$ & $\begin{array}{l}\text { CR855934 } \\
\text { CR855933 } \\
\text { CR855927 } \\
\text { CR855926 } \\
\text { CR855925 } \\
\text { CR855924 } \\
\text { CR855923 } \\
\text { CR855922 } \\
\text { CR855921 } \\
\text { CR855920 } \\
\text { CR855914 } \\
\text { CR855913 } \\
\text { CR855919 } \\
\text { CR855918 } \\
\text { CR855917 } \\
\text { CR855916 } \\
\text { CR855915 } \\
\text { CR855932 } \\
\text { CR855931 } \\
\text { CR855930 } \\
\text { CR855929 } \\
\text { CR855928 } \\
\text { gi124392812 } \\
\text { gi124423260 } \\
\text { gi124414298 }\end{array}$ & $\begin{array}{l}\text { GSPATG00000949001 } \\
\text { GSPATG00008763001 } \\
\text { GSPATG00028086001 } \\
\text { GSPATG00014156001 } \\
\text { GSPATG00026578001 } \\
\text { GSPATG00023985001 } \\
\text { GSPATG00032096001 } \\
\text { GSPATG00028268001 } \\
\text { GSPATG00031597001 } \\
\text { GSPATG00025417001 } \\
\text { GSPATG00001528001 } \\
\text { GSPATG00008137001 } \\
\text { GSPATG00005608001 } \\
\text { GSPATG00030543001 } \\
\text { GSPATG00004175001 } \\
\text { GSPATG00022869001 } \\
\text { GSPATG00020451001 } \\
\text { GSPATG00018824001 } \\
\text { GSPATG00018363001 } \\
\text { GSPATG00013471001 } \\
\text { GSPATG00005704001 } \\
\text { GSPATG00016757001 } \\
\text { GSPATG00004714001 } \\
\text { GSPATG00003023001 } \\
\text { GSPATG00014358001 } \\
\text { GSPATG00028565001 }\end{array}$ & $\begin{array}{l}\text { present } \\
\text { present } \\
\text { present } \\
\text { present } \\
\text { present } \\
\text { present } \\
\text { present } \\
\text { present } \\
\text { present } \\
\text { present } \\
\text { present } \\
\text { present } \\
\text { present } \\
\text { present } \\
\text { present } \\
\text { present } \\
\text { present } \\
\text { present } \\
\text { present } \\
\text { present } \\
\text { present } \\
\text { absent } \\
\text { present } \\
\text { present } \\
\text { present } \\
\text { absent }\end{array}$ & $\begin{array}{l}\text { present } \\
\text { present } \\
\text { present } \\
\text { present } \\
\text { present } \\
\text { present } \\
\text { present } \\
\text { present } \\
\text { present } \\
\text { present } \\
\text { present } \\
\text { present } \\
\text { present } \\
\text { present } \\
\text { present } \\
\text { present } \\
\text { present } \\
\text { present } \\
\text { present } \\
\text { present } \\
\text { present } \\
\text { absent } \\
\text { present } \\
\text { present } \\
\text { present } \\
\text { two present }\end{array}$ & $\begin{array}{l}Q \\
Q \\
Q \\
Q \\
Q \\
Q \\
Q \\
Q \\
Q \\
Q \\
Q \\
Q \\
Q \\
Q \\
Q \\
Q \\
Q \\
Q \\
Q \\
A \\
Q \\
\bar{Q} \\
Q \\
Q \\
Q / Q\end{array}$ & $\begin{array}{l}\text { yes/Qa subgroup } \\
\text { yes/Qa subgroup } \\
\text { yes/Qa subgroup } \\
\text { yes/Qa subgroup } \\
\text { yes/Qa subgroup } \\
\text { yes/Qa subgroup } \\
\text { yes/Qa subgroup } \\
\text { yes/Qa subgroup } \\
\text { yes/Qa subgroup, no syntaxin domain } \\
\text { yes/Qa subgroup, no syntaxin domain } \\
\text { yes/Qa subgroup } \\
\text { yes/Qa subgroup, no syntaxin domain } \\
\text { yes/Qa subgroup, no syntaxin domain } \\
\text { yes/Qa subgroup, no syntaxin domain } \\
\text { yes/Qa subgroup, no syntaxin domain } \\
\text { yes/Qa subgroup, no syntaxin domain } \\
\text { yes/Qa subgroup, no syntaxin domain } \\
\text { yes/Qa subgroup, no syntaxin domain } \\
\text { yes/Qa subgroup, no syntaxin domain } \\
\text { yes/Qa subgroup } \\
\text { yes/Qa subgroup, no syntaxin domain } \\
\text { fragmented/pseudogene? } \\
\text { yes/Qc subgroup, no syntaxin domain } \\
\text { yes/Qc subgroup, no syntaxin domain } \\
\text { yes/Qa subgroup, no syntaxin domain } \\
\text { yes/Qb/c subgroup }\end{array}$ \\
\hline
\end{tabular}


previously with different genes of Paramecium and finally extended to a useful silencing method (Galvani and Sperling 2002; Meyer and Cohen 1999; Ruiz et al. 1998).

A combination of all these methodologies allowed us to explore the identity, localization and function of SNAREs in Paramecium.

\section{The SNARE Repertoire of $\boldsymbol{P}$ tetraurelia}

In Paramecium, most Q-SNARE are of the Qa type (PtSyx 1 to 13), two (PtSyx14 and PtSyx15) are of the Qc type (Kissmehl et al. 2007). Only one is a $\mathrm{Qb} / \mathrm{c}$, i.e., SNAP-25-like protein (SNAP-26-LP) (Schilde et al. 2008). Also PtSNAP-25-LP contains two SNARE domains yet no indication of any C-terminal lipid modification for membrane insertion could be found (Schilde et al. 2008). This unusual situation is known also from some homologous proteins in higher eukaryotes (Holt et al. 2006). For some deviations of amino acid residues in the zero-layer of Paramecium SNAREs, see Tables 1 and 2. Such situations are rare in t-/Q-SNAREs (PtSys 11; Table 2), but are much more frequent in R-SNAREs (PtSyb8, PtSyb9, PtSyb10, and PtSyb11; Table 1). Remarkable is the occurrence of the same aberrant amino acid type in the zero-layer of ohnolog pairs. Other unorthodox features are the absence of a SNARE domain and consequently of a typical zero-layer (PtSyb4, PtSyb5, disregarding questionable SNAREs or likely pseudogenes, PtSyx 13 and PtSyb12, in Tables 1 and 2). When present as doublets, again both ohnologs share this feature (PtSyb4).

To recall, NSF is a SNARE-specific chaperone (Whiteheart et al. 2001) engaged in disentangling SNAREs after fusion (Littleton et al. 2001) and/or in arranging them into a fusogenic complex (Ungermann and Langosch 2005). In Paramecium we have combined NSF gene silencing experiments in the temperature-sensitive mutant, nd9, with a temperature shift (normally allowing the acquirement of exocytosis competence) with exocytosis stimulation in time-sequence series. This has provided more stringent evidence than available from most other systems that NSF can serve the assembly of SNARE complexes (Froissard et al. 2002; Kissmehl et al. 2002), rather than merely SNARE disentangling after fusion - the standard view in most, though not all, published work.

To understand SNARE function, localization and gene silencing are of paramount importance.
Expression as GFP-fusions was preferably performed with GFP at the carboxy-terminus. GFP at the amino-terminus may interfere with intracellular transport, as we have experienced with PtSyb10, for example (Schilde et al. 2010). In most cases, GFP localization studies have been complemented by immuno-localization of the endogenous protein. Eventually overexpression as a GFPfusion protein, followed by immune-gold EM labelling with anti-GFP antibodies was advised to achieve sufficient sensitivity.

Gene silencing is normally possible by the use of silencing constructs ( $\mathrm{PPDx}$ vector) for injection into the macronucleus or for amplification in RNaselll-deficient $E$. coli (strain HT115) for silencing by feeding (Galvani and Sperling 2002; Ruiz et al. 1998). The release of dsRNA activates a posttranslational homology-dependent silencing mechanism which is similar to - though not identical with - the mechanism responsible for macronuclear (maternal) control of modified gene transmission from the micronucleus to a newly forming macronucleus (Duharcourt et al. 2009).

Is it possible to determine the number of SNAREs in Paramecium? To appreciate the number of genes and translation products of SNARE in Paramecium one has to consider several aspects. Many premature automatic annotations proved unreliable, not the least because introns cannot be predicted with sufficient reliability. Moreover, to derive from Tables 1 and 2 the sum of "real" PtSNARE genes one has to consider that some, though very few, may be non-functional genes or pseudogenes. Examples are Ptsyx 13, Ptsyb6-2 and possibly Ptsyb12, whereas Ptsyb6-1 appears to be functional. Omitting questionable forms (truncated forms, potential pseudogenes, etc.) we would end up with 44 SNAREs in $P$. tetraurelia, encompassing 19 synaptobrevin-like and 25 syntaxin-like forms. Among them are 5 singletons and 14 doublets ( 7 ohnolog subfamilies) in the synaptobrevin family vs. 5 singletons and 20 doublets (10 ohnolog subfamilies) in the syntaxin family. On this basis, an estimation would yield a number of "functionally diversified" SNAREs in $P$. tetraurelia, according to our current state of knowledge, of 27 , represented by 12 PtSyb and 15 PtSyx forms. As outlined below, the real number of "functionally diversified" PtSNAREs is probably higher, but the following reasons make it difficult to pinpoint the precise number.

As mentioned, a recent WGD (Aury et al. 2006) has doubled most of the PISNARE genes. For instance, most of the Ptsyb (Schilde et al. 2006, 
2010) and Ptsyx (Kissmehl et al. 2007) genes occur as duplicates/ohnologs, rather than as singletons. This is considered important to estimate the number of "functionally diversified" SNAREs as an indicator of evolutionary progress. To give an example, when inspected in more detail, the genes of Ptsyx 1-1 and Ptsyx 1-2 vary by only $23.1 \%$ of the nucleotides, the respective proteins by $32.1 \%$ of the amino acids. However, these values vary widely for the different PtSNARE types. In Paramecium, the difference between the various ohnologs of syntaxin varies between 6.9 (Ptsyx14) and 60.1\% (Ptsyx8) on a nucleotide basis and between 5.0 (PtSyx2) and 59.8\% (PtSyx8) on an amino acid level (Kissmehl et al. 2007). For synaptobrevins this range is between 11.1 (Ptsyb6) and $37.6 \%$ (Ptsyb9) for nucleotides; on the level of amino acids the difference is between 3.4 (PtSyb7) and 35.4\% (Schilde et al. 2006) or $40.9 \%$ (Schilde et al. 2010), respectively, both values estimated for PtSyb4 (the values for amino acids varying depending on the evaluation criteria applied). Recall that there are also subfamilies represented by only one form, e.g., the singletons of Ptsyb and Ptsyx listed in Tables 1 and 2 , in addition to the single gene for PtSNAP25-LP (Schilde et al. 2008) and for VAMP741 (Schilde et al. 2010) (not further analyzed). Also note that the silencing experiments that target one out of the two ohnologs frequently inhibited the functions of the organelles where this has been localized.

May one derive from this that ohnologs simply serve gene amplification? For the following reasons this has not been strictly shown as yet for PtSNARE ohnolog pairs. On one hand, for silencing, we mainly have used widely identical stretches of the nucleotide sequence common to both ohnologs. On the other hand localization was generally done with a GFP-fusion protein of one ohnolog only, whereas antibody localizations necessarily were less stringent, as antibodies were, for reasons of economy, designed to recognize both forms. Thus, the limit between "functionally diversified" and "functionally equivalent" PTSNARE pairs cannot be indicated with any sufficient clarity. Assuming the limit of $85 \%$ base pair identify for selective gene silencing outlined above and taking into account the number of singletons, one may derive the occurrence of the following number of "functionally diversified" SNARES: $\sim 21$ syntaxin-like, one SNAP-25-LP and $\sim 19$ synaptobrevin-like PtSNAREs (disregarding degenerate forms mentioned above and VAMP741 which is not listed in Table 1 because of lack of further information). This would amount to a total of $\sim 40$ "functionally diversified" PtSNAREs. However, consider that these values are only estimates based on the corollaries indicated.

In addition one has to consider the aberrant structure of some of the Paramecium SNAREs (as specified above). In brief, this can concern the absence of a trans-membrane domain, of a SNARE domain, or of a syntaxin domain (for examples, see Tables 1 and 2). This has not been taken into account as negative characteristics for the estimation of "functionally diversified" SNAREs, above, for the following reasons. (i) There are examples of SNAREs without a transmembrane domain also in other organisms where they can nevertheless function in vesicle interactions, though not in fusion (Thorngren et al. 2004). Therefore, this situation may not be restricted to SNAP-25 and related forms (which are established SNAREs), but this may also occur with other SNAREs. (ii) Furthermore, we know that aberrant amino acids in the zero-layer are not so rare and frequently without major functional consequences (Fasshauer et al. 1998; Graf et al. 2005).

In reality the number of PtSNAREs may be still higher than we have specified above when supplemented by further data from a SNARE database; it may then amount to $\sim 70$ (Kienle et al. 2009; Kloepper et al. 2007). However, expression, topological and functional analyses would still have to be performed for any additional PtSNAREs. We have included in our list one such example, i.e., PtSyb12 which was predicted as a SNARE based on overall sequence similarity.

\section{Overall Intracellular Topology of SNAREs in Paramecium}

The distribution of synaptobrevin- and syntaxinlike SNAREs as well as of SNAP-25-LP in $P$. tetraurelia is illustrated in Figures 5-7. The designations assigned to the different SNARE molecules are not necessarily congruent with those in mammalian cells, particularly with the synaptobrevin-like molecules. Furthermore, the assignment to specific membranes achieved does not preclude the occurrence also at other sites, not only because SNAREs have to travel before they reach their final "homing" membrane, but also because of restrictions in detectability when occurring in small numbers or at low local concentrations, be it even at a specific site.

Six or seven PtSNARE subfamilies are engaged in trafficking along the Endoplasmic Reticulum 


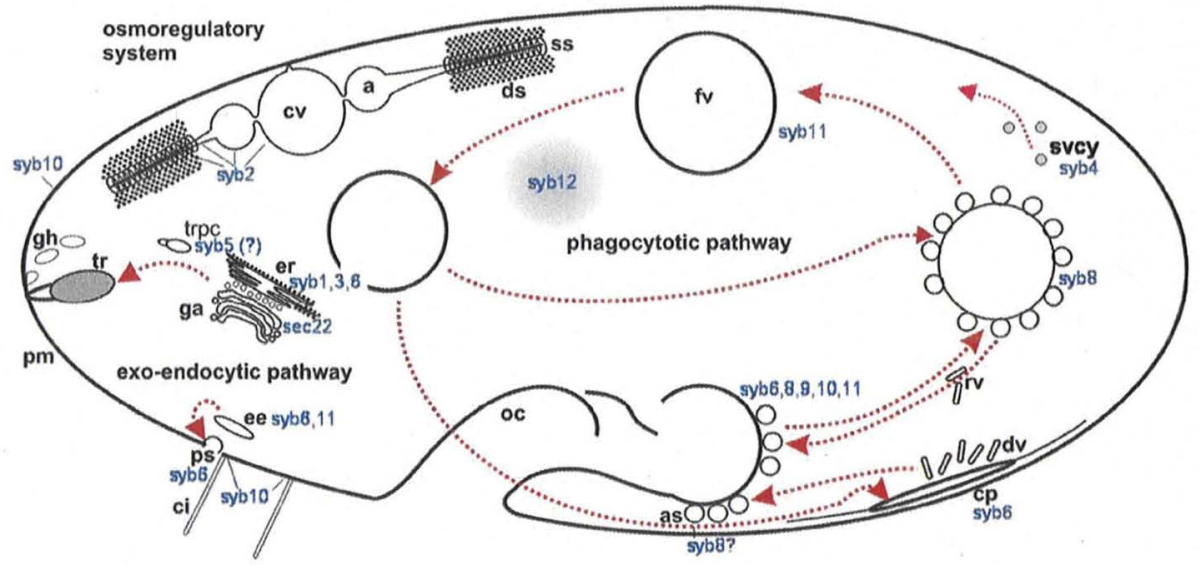

Figure 5. Intracellular distribution of PtSyb paralogs in a Paramecium cell. The respective SNAREs are assigned to the labeled structures (abbreviations as in Fig. 2). Note that PtSyb11 is seen on some of the advanced food vacuoles. The scheme indicates cytosolic localization of PtSyb12, but does not contain autophago(lyso)somes and the lysosomal system proper, as well as the ill-defined populations of small vesicles around the oral cavity. For further comments, see text.

Based on data from Schilde et al. $(2006,2010)$ and Kissmehl et al. (2007).

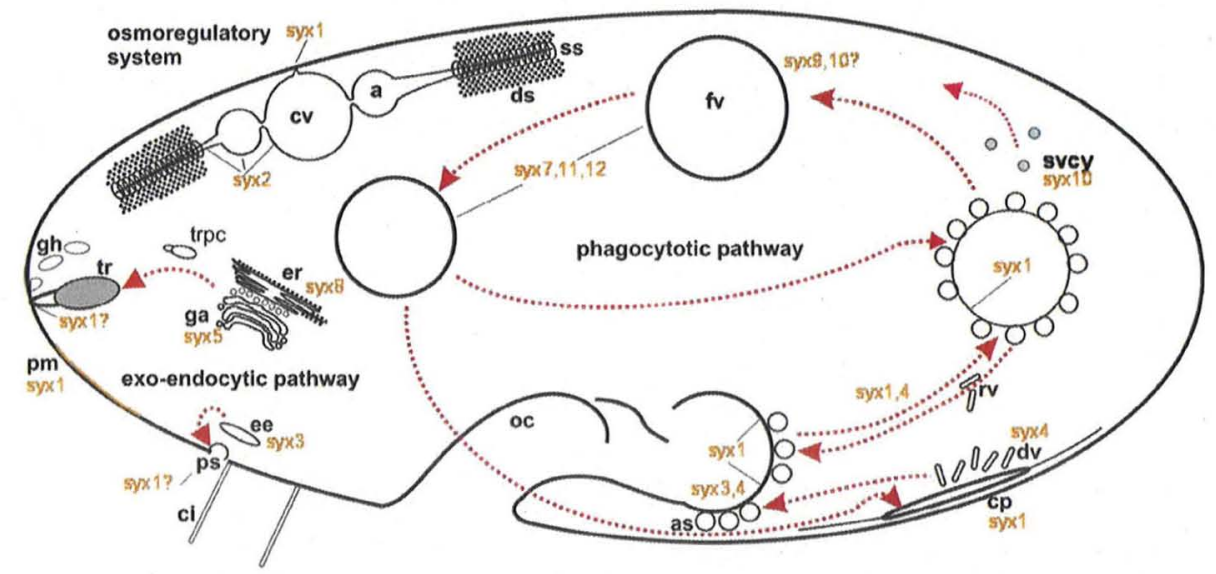

Figure 6. Intracellular distribution of PtSyx paralogs in a Paramecium cell. For abbreviations, see Figure 2 and for further comments, see text.

Based on data from Kissmehl et al. (2007).

(ER)/Golgi pathway, namely Syb1, Syb3, Syb10 (?), Sec22, Syx5, Syx8, and SNAP-25-LP. From the ER, vesicles are escorted by the R-SNARE Sec22 to the Golgi apparatus in other cells (Mancias and Goldberg 2007), as is the case with PtSec22 (Kissmehl et al. 2007), also a longin-type R-SNARE. PtSyx5 associates with the Golgi apparatus, whose R-SNAREs are not known as yet.

No definite SNARE can be assigned as yet to trichocyst precursor vesicles which have to fuse to larger, mature organelles capable of docking and exocytosis (Gautier et al. 1994). There is some circumstantial evidence, however, for the presence of PtSyb5 in these organelles since overexpression as GFP-fusion protein causes irregularly-shaped organelles with immuno-gold labelling using anti-GFP antibodies (Schilde et al. 2010).

The high number of SNAREs in a Paramecium cell, in fact, appears necessary to "serve" all of the multiple and complicated vesicular trafficking 


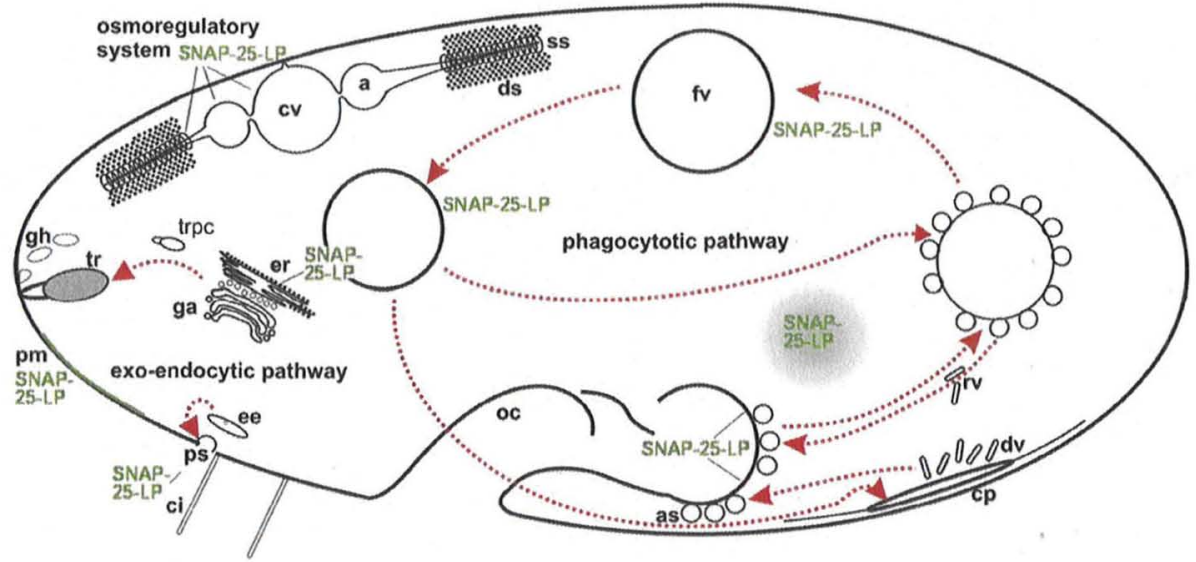

Figure 7. Intracellular distribution of PtSNAP-25-LP in a Paramecium cell. The green background indicates considerable cytosolic localization in unbound form. Some minor details may not have been recognized on this general background. For abbreviations and further comments, see Figure 2 and text.

Based on data from Schilde et al. (2008).

pathways (Figs 6 and 7). Three subfamilies participate in endocytosis via "parasomal sacs" (clathrin-coated pits)/early endosomes, i.e., Syb 11, Syx3, and SNAP-25-LP. Twelve SNARE subfamilies (frequently with ohnologs), i.e., Syb8, Syb9, Syb10, Syb11, Syx 1, Syx4, Syx7, Syx9, Sys10 (?), Syx11, Syx12, and SNAP-25-LP, are engaged alone in the phago-/lysosomal system including membrane recycling. Some of these SNAREs, i.e., PtSyb8 PtSyb9 and PtSyb10 (the latter also occurring at the periciliary cell membrane; see below), are associated with the cytoskeleton organizing the oral cavity which contains a plethora of small vesicles attached (for more details, see below). Therefore one is tempted to assume that some of the vesicles are functionally more diversified than one may guess from their ultrastructure. Small non-acidic PtSyb4positive vesicles circulate in the cyclosis stream and also await further identification.

When expressed as GFP-fusion proteins, SNAREs from several subfamilies occur in the contractile vacuole complex, although the number is much smaller when the endogenous PtSNAREs are visualized by antibodies (Kissmehl et al. 2007; Schilde et al. 2006). This suggests that the contractile vacuole complex unexpectedly participates intensely in vesicle trafficking, well beyond the overt pumping and extrusion cycle, as discussed in more detail below.

PtSyx1 has been localized as a GFP-fusion protein and, by subsequent EM immunocytochemistry, to the somatic (non-ciliary) cell membrane where it is scattered all over (Kissmehl et al. 2007). Though it is not visibly enriched at preformed trichocyst docking/exocytosis sites Ptsyx1 silencing greatly inhibits exocytosis. Its scattered occurrence in the cell membrane may also serve inconspicuous constitutive exocytosis for which previously the parasomal sacs (in between clathrin coat-mediated endocytotic activity) have been exclusively implicated (Capdeville 2000; Flötenmeyer et al. 1999). Occurrence of such sites outside parasomal sacs is supported by the observation of numerous small vesicles attached at the cell membrane when NSF is silenced (Schilde et al. 2010). This procedure can "freeze" such cryptic sites of vesicle delivery and, thus, make them visible (Kissmehl et al. 2002), whereas this process would normally be so fast as to escape detection.

We observed the enrichment of PtSyb10 labelling at the transition of the ciliary basis to the nonciliary ("somatic") cell membrane when expressed with a carboxy-terminal GFP-tag and visualized by anti-GFP antibodies/gold-labeling at the EM level (Schilde et al. 2010). As generally assumed, biogenesis of cilia involves vesicle delivery close to the ciliary basis and fusion for further transport as "rafts" within the ciliary membrane (Rosenbaum and Witman 2002). However, SNAREs involved in that process are largely unknown, with very few exceptions. The functional significance of the PtSyb10 localization around ciliary bases has been analyzed by tandem silencing of both ohnolog genes. In that case, depolarization-induced 
rotation of cells during ciliary beat reversal was significantly slowed down (Schilde et al. 2010).

\section{Classical Endo-/Phago-/Lysosomal and Membrane Recycling Systems and their Molecular Equivalents in Paramecium}

In ciliates, phagosomes are formed adjacent to the lowest part of the oral cavity, the cytopharynx. It first looks like a local invagination of the cell membrane, but in reality considerable membrane material is delivered by several different types of vesicles endowed with their specific SNAREs (see below). A population of vesicles, probably of mixed type but of unsettled origin, is seen in video-microscopy to travel along the "postoral fiber" microtubules in parallel orientation to the cytopharynx (Ishida et al. 2001). Shortly after pinching off, numerous acidosomes fuse with the new phagosome (Allen and Fok 1983). Acidosomes are considered late endosomes (Allen et al. 1993). This is followed by fusion with lysosomes, to yield a mature phagolysosome ("food vacuole", "digestive vacuole"). Parts of its membrane are recycled in the form of "discoidal vesicles" back to the nascent food vacuole, not only from the mature food vacuole (Allen et al. 1995) but also from the spent vacuole after undigested material has been released by exocytosis at the cytoproct (Schroeder et al. 1990). On the way through the Paramecium cell a food vacuole receives additional vesicle input from "parasomal sacs" (clathrin-coated pits) via "terminal cisternae" (early endosomes) (Allen et al. 1992) as well as from piecemeal delivery from trichocyst "ghosts" once exocytosis has been stimulated (Lüthe et al. 1986). All this has been repeatedly summarized (Allen and Fok 2000; Fok and Allen 1990).

This complex interaction scheme in the endo-/ phago-/lysosomal system of a Paramecium cell is reflected by the multitude of SNAREs in this system. PtSyx1, the dominant SNARE of the somatic cell membrane forms part of the nascent food vacuole membrane where it is still detectable early on after pinching off. The presence of PtSyx 1 indicates that there, in fact, also occurs some input from the cell membrane into the phagosomal membrane. Furthermore it receives PtSyx4 from recycling vesicles (Kissmehl et al. 2007). Further on, PtSyx 1 is exchanged for PtSyx7, 11 and 12 on the way through the cell, but PtSyx 9 and 10 also contribute temporarily. During the overall travel through the cell PtSyx 1, 4, 7, 9, 10, 11 and 12 show up in the phago-/lysosomal system. This is complemented by syntaxins from the Golgi apparatus (PtSyx5) and from early endosomes (PtSyx3). Possibly some more Qa-SNAREs may occur but remain undetectable when parts of the system undergo rapid turnover. PtSNAP-25-LP accompanies food vacuoles only after acidosomal membranes had been removed for recycling (Schilde et al. 2008).

Less is known about the participation of $v-/ R$ SNAREs in membrane trafficking during cyclosis. Acidosomes probably contribute PtSyb8, PtSyb9 and PtSyb10 (Schilde et al. 2010). PtSyb9 and PtSyb10 are associated with the small vesicles travelling along microtubules parallel to the cytopharynx surface (see above); as stated, these probably also provide membranes for food vacuole formation (Ishida et al. 2001). PtSyb8 is recognized still after some travelling time on the phago(lyso)some membrane (Schilde et al. 2010).

To complete the list of SNARE input into the endo-/phago-/lysosomal system in Paramecium, one has to add the following sites of distinct vesicle labelling. Parasomal sacs contain PtSyb6 (Schilde et al. 2006) and PtSNAP-25-LP (Schilde et al. 2008). Early endosomes ("terminal cisternae") are endowed with PtSyb6 (Schilde et al. 2006) and PtSyb11 (Schilde et al. 2010) as well as with PtSyx3 (Kissmehl et al. 2007). The cytoproct contains PtSyb6 (Schilde et al. 2006). It remains to be determined whether PtSyx1 serves as a $t-/ Q-S N A R E$ also at this site of the cell membrane.

All this makes a considerable number of possible SNARE combinations that can keep up with the multiple membrane fusion and fission sites in this dynamically interacting and intriguingly complicated part of a Paramecium cell. It may actually be the main "reason" why in these cells SNAREs have become so highly diversified during evolution.

\section{Contractile Vacuole Complex - A Classical View and Molecular Equivalents}

This complex organelle, also called the osmoregulatory system, is present in duplicate in a Paramecium cell. Each one is made up of a contractile vacuole and $\sim 6$ emanating "radial (collecting) canals" that are permanently connected to the "spongiome" (Allen and Naitoh 2002). The spongiome is a widely branched tubular system, the distal part of which is "decorated", as visible in the EM, by $\mathrm{H}^{+}$-ATPase/pump molecules (Fok et al. 2002). 
Water chemiosmotically sequestered by the $\Delta \mathrm{H}^{+}$ moves from the smooth part of the spongiome to the collecting ducts and then into the vacuole for expulsion, with every systole, by exocytosis at preformed sites (Allen and Naitoh 2002). Other sites of cyclic membrane fusion are the connections of the canals with the vacuole, as found by surface capacitance measurements (Tominaga et al. 1998).

We could selectively immuno-label these conspicuous membrane interaction sites, i.e., vacuole expulsion site ("porus") and vacuole/canal connections, by anti-NSF antibodies. This required an unusual approach, however. Cautiously permeabilized (surviving) cells were exposed to the NSF inhibitor, $\mathrm{N}$-ethylmaleimide, and to the non-hydrolyzable ATP analog, ATP- $\gamma$-S (Kissmehl et al. 2002). This approach is based on the following rationale. As an AAA-ATPase-type SNARE chaperone, NSF normally detaches itself from membrane-to-membrane interaction sites after having established SNARE-mediated contacts for fusion (Whiteheart et al. 2001). Inhibition of the ATPase activity of NSF, required to exert its chaperone function, by $\mathrm{N}$-ethylmaleimide and ATP- $\gamma-\mathrm{S}$ can evidently "freeze" such short-lived situations.

Surprisingly NSF inhibition in surviving permeabilized cells, when subjected to anti-NSF antibody immuno-fluorescence labelling, also resulted in labelling of vacuole, canals and (smooth) spongiome. Their labelling with anti-NSF antibodies was much less intense, though (Kissmehl et al. 2002). By immuno-labelling using antibodies against GFP in SNARE-GFP fusion proteins, we could localize in normal (not NSF silenced) cells PtSyx2, 14 and 15 and PtSyb2, 6 and 9, as well as PtSNAP-25-LP over the membranes of the contractile vacuole complex at the light microscope level, with the exception of the decorated spongiome (Kissmehl et al. 2007; Schilde et al. $2006,2008,2010)$. However, when immunolocalized by anti-SNARE antibodies without previous overexpression as GFP fusion proteins, only PtSyx2 and PtSyb2 were localized exclusively to the contractile vacuole complex (Kissmehl et al. 2007; Schilde et al. 2006). In contrast to NSF labelling under the inhibitory conditions described above (N-ethylmaleimide + ATP- $\gamma$-S), no hot spots became visible with anti-SNARE antibodies, but labelling was rather homogenous. This is precisely what one would expect under the respective conditions.

What function may SNAREs exert in this complex organelle outside overt sites of cyclic membrane fusion? Three hypotheses may now be formulated: (i) SNAREs may serve steady-state biogenesis, i.e., organelle turnover by membrane delivery. However, nobody has ever recognized any overt vesicle transport. (ii) A trans-complex SNAREs from adjacent membranes could maintain the tubules of the smooth spongiome in their typical, densely packed arrangement. (iii) SNAREs could serve the rearrangement of membranes during systole/diastole cycles.

Hypothesis (i) is supported by EM analyses revealing the emaciation of the spongiome during ongoing NSF silencing (unpublished observations). Support also comes from the fact that, in mammalian systems, some of the SNAREs travel together with some of the $\mathrm{H}^{+}$-ATPase subunits from the ER to the Golgi by vesicles and beyond (Schwartz et al. 2007). Additional support comes from yeast cells. Here, in a first step, $\mathrm{H}^{+}$-ATPase components are enabled to escape to the Golgi apparatus by an assembly factor, Vma21p, which binds to COPIl-type coats and thus induces budding of vesicles for transport to the Golgi apparatus (Malkus et al. 2004). In a second step, ER/Golgi SNAREs of the types Sec22 and syntaxin may be included in such complexes (Mossessova et al. 2003) by interaction of the longin domain of Sec22 (Liu et al. 2004) via a conformational motif (Mancias and Goldberg 2007). All this assigns a role to Sec22 as a selecting component, together with the likely function as a fusion regulator. Remarkably Sec22 is present in ER-rich domains also in Paramecium (Kissmehl et al. 2007). A similar scenario, corresponding to hypothesis (i), may also hold true for the biogenesis of the contractile vacuole complex of Paramecium, but definite proof is still lacking at this time. In contrast, we do not favour hypothesis (ii), although not rejectable, as this would not explain the occurrence of SNAREs also outside the smooth spongiome. Hypothesis (iii) is also plausible - although less likely than hypothesis (i). In fact, in diastole the vacuole and the canal membranes collapse to interconnected tubules (Allen and Fok 1988); this could require reconstitution, possibly by fusion events. Such transformation is required to explain that, in the light microscope, during systole vacuole size apparently becomes smaller. However, hypothesis (iii) would not be applicable to the smooth spongiome where SNAREs are also found.

Since silencing of either Ptsyx2 or Ptsyb2 in pilot experiments affects structure and function of the contractile vacuole system (unpublished observations) the SNAREs in this organelle can reasonably be assumed to contribute to ongoing 


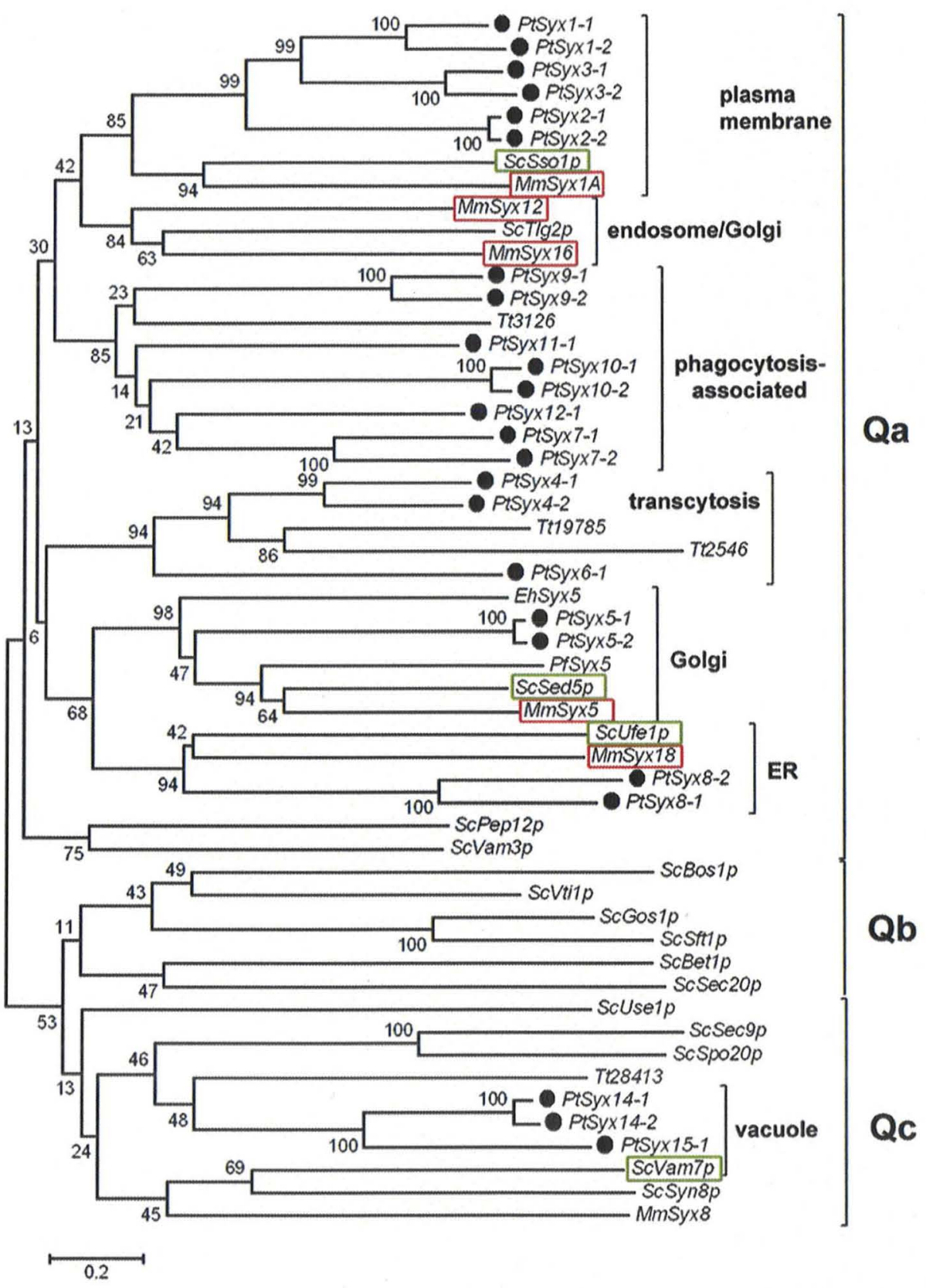

Figure 8. Evolutionary and topological relationships of $P$. tetraurelia syntaxins (PtSyx) with syntaxins from other organisms (neighbor joining tree). Note grouping in Qa-, Qb- and Qc-SNAREs, whereby Qa-types predominate in Paramecium, Qb having not been found. Species abbreviations: Eh=Entamoeba histolytica, $\mathrm{Hs}=$ Homo sapiens, Mm=Mus musculus, $\mathrm{Pf}=$ Plasmodium falciparum, $\mathrm{Sc}=$ Saccharomyces cerevisiae, $\mathrm{Tt}=$ Tetrahymena thermophila. Coloured boxes indicate SNAREs with characteristic localization in other species. For details on methodology and sources of sequences, see Kissmehl et al. (2007); figure reproduced with permission.

biogenesis (hypothesis [i]). As mentioned, in the contractile vacuole complex the hot spots of the dynamic, cyclic function, i.e., the vacuole/cell membrane and the vacuole/radial canal interaction sites, definitely require the activity of SNAREs (again hypothesis [i]). This kind of "static" 
trafficking may be compared with a soldier's "marking time".

\section{Localization Seen in an Evolutionary Context}

Among PtSNAREs, it was possible specifically with syntaxins to compare established homologs in the context of their intracellular localization up to man (Kissmehl et al. 2007), as shown in Figure 8. In $P$. tetraurelia this concerns Qa-type PtSyx, Qb being absent, $Q c$ rare, and $Q b / c$ represented by only one member, SNAP-25-LP (Table 2). One aspect one can see in Figure 8 is that some PtSNARE groups have no counterparts in higher eukaryotes. Another aspect is a relationship of some PtSNAREs with some SNAREs in Tetrahymena thermophila (as far as data are available). Such groups include PtSyx4 and PtSyx6, both with distant relatives in $T$. thermophila. In P. tetraurelia, according to Figure 8 , these SNAREs seem to be restricted to a kind of transcytotic recycling pathway from the cytoproct as well as from maturing phago(lyso)somes to the newly forming phagosome. (For PtSyx6 this is hypothetically derived from the context in Figure 8 as we could not achieve localization by any of the GFP-fusions). Even more interesting is the localization (Fig. 6) and grouping (Fig. 8) of the large number of unique PtSNAREs encompassing PtSyx7, PtSyx9, PtSyx10, PtSyx11, and PtSyx12 which all participate in the phago(lyso)somal system. All this rather clearly suggests molecular diversification in the context of increased phago(lyso)somal trafficking including extensive membrane recycling phenomena.

\section{Comparison with Other Organisms}

We found many SNAREs with "orthodox" features in $P$. tetraurelia which is practically the only ciliate and one of a few protozoans analyzed until now with some consistency. We have identified so far 44 genes encoding bona fide SNAREs, while some sequences are questionable; see above for details. Since there are numerous ohnolog pairs with high similarity, whereas some others are more widely different, one may conclude that there may be $\sim 40$ "functionally diversified" PtSNAREs. Some are singletons, thus reflecting the tendency to eliminate one of the ohnologs during evolution (Byrne and Wolfe 2005) also in Paramecium (Aury et al. 2006). A systematic database search has yielded a number of $\sim 70$ SNARE sequences in $P$. tetraurelia (Kienle et al. 2009; Kloepper et al. 2007) although these have not yet been all identified in detail on the expression level etc. For comparison, evolutionary analysis has suggested that the 'ur-eukaryote' may have been endowed with $\sim 20$, the 'ur-metazoan' with $\sim 30$ SNAREs (Kloepper et al. 2008).

The data obtained with Paramecium can now be compared on one hand with 'higher' eukaryotes and on the other hand with some other protists (inasmuch as comparable, reliable data are available). For instance, Saccharomyces cerevisiae possesses 26, i.e., 7 Qa-, 6 Qb-, 8 Qc- and 5 R-SNAREs (Burri and Lithgow 2004); particularly the fraction of Qa- and of R-SNAREs is small when compared with Paramecium. Arabidopsis thaliana possesses 42 (Lipka et al. 2007) or perhaps 64 (Sanderfoot 2007; therein supplementary material, table) SNARE genes. An estimated number of 39 (Sanderfoot 2007) or 41 SNARE genes is indicated for man (Kloepper et al. 2007). Clearly, Paramecium is far off an early evolutionary eukaryote stage and, thus, seems to have gone independently its own evolutionary way. Most probably this has been enabled by at least two rounds of WGD processes preceding the last WGD and subsequent diversification, whereas the last WGD probably may have contributed little - if anything - to diversification of SNAREs in P. tetraurelia.

In conclusion, alone the mere number of SNAREs in Paramecium would suggest that diversification of SNAREs took place independently in ciliates (strictly only in Paramecium where SNAREs have been sufficiently well analyzed) and in 'higher' eukaryotes. In particular, we see in Figures 6 and 8 diversification of syntaxins within the phago(lyso)somal apparatus and this may be a cause (or a consequence) of molecular diversification (much more than the contractile vacuole system). Unfortunately comparably detailed data from other protists are rare. Earlier claims that protozoa would have a low number of SNAREs were based on parasitic species (Yoshizawa et al. 2006) and, thus, may be selective. For instance, Giardia lamblia contains 17 (Elias et al. 2008) and the malaria causing species, Plasmodium falciparum, 18 SNAREs (Ayong et al. 2007). In this context it should be recalled that the latter, as a member of the phylum Apicomplexa, belongs to the Alveolata, together with ciliates. At first sight the low numbers in the parasitic forms may be attributed to their lifestyle, 
but also in most other unicellular organisms, including algae, the number of SNARE genes is generally considerably lower than in $P$. tetraurelia. Examples are the diatoms, Phaeodactylum tricornutum and Thalassiosira pseudonana, with 19 and 18 SNARE genes, respectively; furthermore the unicellular red alga, Cyanidioschyzon merolae with 16 and the picoplanktonic prasinophyte Ostreococcus tauri with 20 SNARE genes (Sanderfoot 2007). Even the protozoan slime mold, Dictyostelium discoideum, with its complex life cycle is reported to contain only 23 SNARE genes (Sanderfoot 2007). The number of SNARE genes in $P$. tetraurelia also exceeds the 24 found in S. cerevisiae (Burri and Lithgow 2004) which one may consider a minimum for metazoan cell function.

In sum the number of SNAREs in a Paramecium cell is rather high even when compared with some simple metazoans. From all these arguments an evolutionarily interesting aspect emerges: In parallel to their considerable structural complexity, particularly in the vesicle trafficking system, ciliates have diversified concomitantly their SNARE machinery. In other words, there occurred a parallel evolution in the protozoan and in the metazoan organisms in this respect.

\section{Some Major Open Aspects for Future Research}

Beyond SNAREs, in Paramecium just as in 'higher' eukaryotes, the spectrum of proteins interacting during membrane-to-membrane tethering, docking and fusion also includes other key-players, such as "auxiliary" SNARE-associated proteins, small GTPases, $\mathrm{H}^{+}$-ATPase subunits and F-actin and probably many more. Therefore, beyond the questions formulated above, there are many other basic aspects pertinent to an understanding of vesicle trafficking in protozoa in general and in ciliates in particular. Only some of these questions may be listed as paradigmatic examples for future research.

- Which role plays the binding of accessory/ auxiliary proteins to SNAREs (Rizo et al. 2006; Weninger et al. 2008; Wojcik and Brose 2007)? In fact, some of these proteins are also found in the Paramecium cell. This includes Munc18/ Sec1 and $\alpha$-SNAP (unpublished data; annotations by Roland Kissmehl, this laboratory).

- It has been reported for 'higher' eukaryotic cells that calmodulin binds to synaptobrevin (Quetlas et al. 2002). Remarkably, in Paramecium calmodulin is required for assembling exocytosis sites (Kerboeuf et al. 1993) where calmodulin has also been localized by antibody techniques (Momayezi et al. 1986). Molecular details are not known as yet.

- Which monomeric GTP-binding proteins (GTPases) participate, together with their regulators, in vesicle trafficking, as amply documented for many cell types (Bonifacino and Glick 2004; Grosshans et al. 2006; Novick and Zerial 1997)? With T. thermophila a comprehensive attempt along these lines is currently performed by the group of Aaron Turkewitz (University of Chicago).

- Also missing is a thorough investigation of coatamer proteins involved in vesicle budding, as shown for higher eukaryotes (Bonifacino and Glick 2004; Maranda et al. 2001). First attempts toward a systematic analysis in protists have been presented by Dacks and Field (2007).

- Does acidification of the lumen of some of the organelles produce a trans-membrane signal through a conformational change of the multiheteromeric $\mathrm{H}^{+}$-ATPase molecule which then can bind different GTPases and their modulators on the cytosolic side? This has been reported for yeast (Hurtado-Lorenzo et al. 2006) for differential vesicle recognition/targeting? The exchange of components we described in $P$. tetraurelia during the acidification/neutralization cycle of the phago(lyso)some in the course of cyclosis (Wassmer et al. 2009) points in this direction.

- To what extent interact SNAREs with coatamer proteins, as reported for other cells (Mossessova et al. 2003)?

- Is there an interaction of $\mathrm{H}^{+}$-ATPase subunits with SNAREs required to direct vesicle trafficking? For instance, in kidney-collecting ducts the $\mathrm{H}^{+}$-ATPase V1-part and syntaxin 1 co-migrate to the cell membrane (Schwartz et al. 2007) and the $\mathrm{H}^{+}$-ATPase in turn was shown to bind by its C-subunit to F-actin, also shown for metazoan cells (Beyenbach and Wieczorek 2006). Shortly, to what extent do SNAREs cooperate with the proton pump and with $\mathrm{F}$-actin during vesicle trafficking?

- Which is, in ciliates, the $\mathrm{Ca}^{2+}$-sensor in stimulated SNARE-mediated membrane fusion? Normally this is synaptotagmin, endowed with two C2-domains, but in Paramecium such a protein with two C2-domains has not been found in the database. Is the situation similar to that in 
plants, where similar proteins with deviating numbers of C2-domains occur (Craxton 2007)?

- Which SNAREs are engaged in biogenesis of cilia? This is a largely unexplored field, also in metazoans.

All this, together with the many open details concerning SNAREs, is an ample field for future research. The goal will be to put previous insights obtained with the tools of classical cell biology onto a molecular foundation, using the new tools recently available.

\section{Acknowledgements}

The author thanks all former collaborators, in particular Dr. R. Kissmehl for his great efforts in developing the Paramecium SNARE project, as well as Drs C. Schilde and I. M. Sehring and all the diploma students involved in this project. Thanks are also due to Drs R. Jahn and D. Fasshauer (Max-Planck-Institute Göttingen) for making the SNARE database accessible and to Drs J. Cohen and $L$. Sperling for establishing and opening early on the Paramecium database as well as for organizing the "Groupement de Recherche Européen" with its numerous international interactions. Particular thanks are also due to Linda Sperling for carefully monitoring this manuscript. The author's work cited herein has been continuously supported by the Deutsche Forschungsgemeinschaft.

\section{Note added in proof}

Beyond SNAREs, the relevance of $\mathrm{H}^{+}$-ATPase and actin isoforms for vesicle trafficking in ciliates has been recently summarized by Plattner 2010 Int. Rev. Cell Mol. Biol. 280:79.

\section{References}

Allen RD, Fok AK (1983) Nonlysosomal vesicles (acidosomes) are involved in phagosome acidification in Paramecium. J Cell Biol 97:566-570

Allen RD, Fok AK (1988) Membrane dynamics of the contractile vacuole complex of Paramecium. J Protozool 35:63-71

Allen RD, Fok AK (2000) Membrane trafficking and processing in Paramecium. Int Rev Cytol 198:277-317

Allen RD, Naitoh Y (2002) Osmoregulation and contractile vacuoles of protozoa. Int Rev Cytol 215:351-394
Allen RD, Ma L, Fok AK (1993) Acidosomes: recipients of multiple sources of membrane and cargo during development and maturation. J Cell Sci 106:411-422

Allen RD, Schroeder CC, Fok AK (1992) Endosomal system of Paramecium: coated pits to early endosomes. J Cell Sci 101:449-461

Allen RD, Bala NP, Ali RF, Nishida DM, Aihara MS, Ishida M, Fok AK (1995) Rapid bulk replacement of acceptor membrane by donor membrane during phagosome to phagoacidosome transformation in Paramecium. J Cell Sci 108:1263-1274

Arnaiz O, Cain S, Cohen J, Sperling L (2007) ParameciumDB: a community resource that integrates the Paramecium tetraurelia genome sequence with genetic data. Nucleic Acids Res 35:D439-D444

Aury J-M, Jaillon O, Duret L, Noel B, Jubin C, Porcel BM, Ségurens $B$, Daubin V, Anthouard $V$, Aiach $N$, Arnaiz $O$, Billaut A, Beisson J, Blanc I, Bouhouche K, Camara F, Duharcourt S, Guigo R, Godendeau D, Katinka M, Keller A-M, Kissmehl R, Klotz C, Koll F, Le Mouel A, Lepère G, Malinsky S, Nowacki M, Nowak J, Plattner H, Poulain J, Ruiz $F$, Serrano $V$, Zagulski $M$, Dessen $P$, Bétermier $M$, Weissenbach J, Scarpelli C, Schächter V, Sperling L, Meyer E, Cohen J, Wincker P (2006) Global trends of whole genome duplications revealed by the genome sequence of the ciliate Paramecium tetraurelia. Nature 444:171-178

Ayong L, Pagnotti G, Barrero Tobon A, Chakrabarti D (2007) Identification of Plasmodium falciparum family of SNAREs. Mol Biochem Parasitol 152:113-122

Beisson J, Lefort-Tran M, Pouphile M, Rossignol M, Satir B (1976) Genetic analysis of membrane differentiation in Paramecium. Freeze-fracture study of the trichocyst cycle in wild-type and mutant strains. J Cell Biol 69:126-143

Bethani I, Lang T, Geumann U, Sieber JJ, Jahn R, Rizzoli SO (2007) The specificity of SNARE pairing in biological membranes is mediated by both proof-reading and spatial segregation. EMBO J 26:3981-3992

Beyenbach KW, Wieczorek H (2006) The V-type $\mathrm{H}^{+}$ATPase: molecular structure and function, physiological roles and regulation. J Exp Biol 209:577-589

Bock JB, Scheller RH (1996) A new syntaxin family member implicated in targeting of intracellular transport vesicles. $J$ Biol Chem 271:17961-17965

Bonifacino JS, Glick BS (2004) The mechanisms of vesicle budding and fusion. Cell 116:153-166

Burri L, Lithgow T (2004) A complete set of SNAREs in yeast. Traffic 5:45-52

Byrne KP, Wolfe KH (2005) The yeast gene order browser: combining curated homology and syntenic context reveals gene fate in polyploid species. Genome Res 15: 1456-1461

Capdeville Y (2000) Paramecium GPI proteins: variability of expression and localization. Protist 151:161-169

Craxton M (2007) Evolutionary genomics of plant genes encoding $\mathrm{N}$-terminal-TM-C2 domain proteins and the similar FAM62 genes and synaptotagmin genes of metazoans. BMC Genomics 8:25910.1186/1471-2164-8-259 
Dacks JB, Field MC (2007) Evolution of the eukaryotic membrane-trafficking system: origin, tempo and mode. J Cell Sci 120:2977-2985

Dessen P, Zagulski M, Gromadka R, Plattner H, Kissmehl R, Meyer E, Bétermier M, Schultz JE, Linder U, Pearlman E, Kung C, Forney J, Satir BH, Van Houten J, Keller A-M, Froissard $\mathbf{M}$, Sperling $\mathbf{L}$, Cohen $\mathbf{J}$ (2001) Paramecium genome survey: a pilot project. Trends Genet 17:306-308

Duharcourt S, Lepère G, Meyer E (2009) Developmental genome rearrangements in ciliates: a natural genomic subtraction mediated by non-coding transcripts. Trends Genet 25:344-350

Elias EV, Quiroga R, Gottig N, Nakanishi H, Nash TE, Neiman A, Lujan HD (2008) Characterization of SNAREs determines the absence of a typical Golgi apparatus in the ancient eukaryote Giardia lamblia. J Biol Chem 283:3599636010

Fasshauer D, Sutton RB, Brunger AT, Jahn R (1998) Conserved structural features of the synaptic fusion complex: SNARE proteins reclassified as Q- and R-SNAREs. Proc Natl Acad Sci USA 95:15781-15786

Filippini F, Rossi V, Galli T, Budillon A, D'Orso $M$, D'Esposito M (2001) Longins: a new evolutionary conserved VAMP family sharing a novel SNARE domain. Trends Blochem Sci 26:407-409

Flötenmeyer M, Momayezi M, Plattner H (1999) Immunolabeling analysis of biogenetic and degradative pathways of cell surface components (glycocalyx) in Paramecium cells. Eur J Cell Biol 78:67-77

Fok AK, Allen RD (1990) The phagosome-lysosome membrane system and its regulation in Paramecium. Int Rev Cytol 123:61-94

Fok AK, Yamauchi K, Ishihara A, Aihara MS, Ishida M, Allen AR (2002) The vacuolar-ATPase of Paramecium multimicronuleatum gene structure of the $B$ subunit and the dynamics of the V-ATPase-rich osmoregulatory membranes. $\checkmark$ Eukaryot Microbiol 49:185-196

Froissard M, Kissmehl R, Dedieu J-C, Gulik-Krzywicki T, Plattner $\mathrm{H}$, Cohen J (2002) $\mathrm{N}$-ethylmaleimide-sensitive factor is required to organize functional exocytotic microdomains in Paramecium. Genetics 161:643-650

Galvani A, Sperling $L$ (2002) RNA interference by feeding in Paramecium. Trends Genet 18:11-12

Gautier M-C, Garreau De Loubresse N, Madeddu L, Sperling L (1994) Evidence for defects in membrane traffic in Paramecium secretory mutants unable to produce functional storage granules. J Cell Biol 124:893-902

Graf CT, Riedel D, Schmitt HD, Jahn R (2005) Identification of functionally interacting SNAREs by using complementary substitution in the conserved $O^{\prime}$ layer. Mol Biol Cell 16: 2263-2274

Grosshans BL, Ortiz D, Novick P (2006) Rabs and their effectors: achieving specificity in membrane traffic. Proc Natl Acad Sci USA 103:11821-11827

Hauser K, Haynes W, Kung C, Plattner H, Kissmehl R (2000) Expression of the green fluorescent protein in Paramecium tetraurelia. Eur J Cell Biol 79:144-149
Haynes WJ, Ling K-Y, Saimi Y, Kung C (1996) Toward cloning by complementation in Paramecium. J Neurogenet 11:81-98

Holt $M$, Varoqueaux $F$, Wiederhold $K$, Takamori S, Urlaub H, Fasshauer D, Jahn R (2006) Identification of SNAP-47, a novel Qbc-SNARE with ubiquitous expression. J Biol Chem 281:17076-17083

Hurtado-Lorenzo A, Skinner L, El Annan J, Futai M, SunWada G-H, Bourgoin S, Casanova J, Wildeman A, Bechoua S, Ausiello DA, Brown D, Marshansky V (2006) $V$-ATPase interacts with ARNO and Arf6 in early endosomes and regulates the protein degradative pathway. Nature Cell Biol 8:124-136

Ishida M, Allen RD, Fok AK (2001) Phagosome formation in Paramecium: roles of somatic and oral cilia and of solid particles as revealed by video microscopy. J Eukaryot Microbiol 48:466-640

Jackson MB, Chapman ER (2006) Fusion pores and fusion machines in $\mathrm{Ca}^{2+}$-triggered exocytosis. Annu Rev Biophys Biomol Struct 35:135-160

Jahn R, Lang T, Südhof TC (2003) Membrane fusion. Cell 112:519-533

Jahn R, Scheller RH (2006) SNAREs - engines for membrane fusion. Nature Rev Mol Cell Biol 7:631-643

Kasai $\mathbf{H}$ (1999) Comparative biology of $\mathrm{Ca}^{2+}$-dependent exocytosis: implications of kinetic diversity for secretory function. Trends Neurosci 22:88-93

Keller A-M, Cohen J (2000) An indexed genomic library for Paramecium complementation cloning. J Eukaryot Microbiol 47:1-6

Kerboeuf D, LeBerre A, Dedieu JC, Cohen J (1993) Calmodulin is essential for assembling links necessary for exocytotic membrane fusion in Paramecium. EMBO $\mathrm{J}$ 12:3385-3390

Kienle N, Kloepper TH, Fasshauer D (2009) Differences in the SNARE evolution of fungi and metazoa. Biochem Soc Trans 37:787-791

Kissmehl R, Froissard M, Plattner H, Momayezi M, Cohen $\mathbf{J}$ (2002) NSF regulates membrane traffic along multiple pathways in Paramecium. J Cell Sci 115:3935-3946

Kissmehl R, Schilde C, Wassmer T, Danzer C, Nühse K, Lutter K, Plattner H (2007) Molecular identification of 26 syntaxin genes and their assignment to the different trafficking pathways in Paramecium. Traffic 8:523-542

Kloepper TH, Kienle CN, Fasshauer D (2007) An elaborate classification of SNARE proteins sheds light on the conservation of the eukaryotic endomembrane system. Mol Biol Cell 18:3463-3471

Kloepper TH, Kienle CN, Fasshauer D (2008) SNAREing the basis of multicellularity: consequences of protein family expansion during evolution. Mol Biol Evol 25:2055-2068

Langkjaer RB, Cliften PF, Johnston M, Piskur J (2003) Yeast genome duplication was followed by asynchronous differentiation of duplicated genes. Nature 421:848-852

Lin RC, Scheller RH (1997) Structural organization of the synaptic exocytosis core complex. Neuron 19:1087-1094 
Lipka V, Kwon C, Panstruga R (2007) SNARE-ware: the role of SNARE-domain proteins in plant cells. Annu Rev Cell Dev Biol 23:147-174

Littleton JT, Barnard RO, Titus SA, Slind J, Chapman ER, Ganetzky B (2001) SNARE-complex disassembly by NSF follows synaptic-vesicle fusion. Proc Natl Acad Sci USA 98:12233-12238

Liu Y, Flanagan JJ, Barlowe C (2004) Sec22p export from the Endoplasmic Reticulum is independent of SNARE pairing J Biol Chem 279:27225-27232

Lüthe H, Plattner H, Haacke B, Walther P, Müller M (1986) Lectin binding sites in Paramecium tetraurelia cells. I. Labeling analysis predominantly of secretory components. Histochemistry 85:365-376

Malkus P, Graham LA, Stevens TH, Schekman R (2004) Role of Vma21p in assembly and transport of the yeast vacuolar ATPase. Mol Biol Cell 15:5075-5091

Malsam J, Kreye S, Söllner TH (2008) Membrane fusion SNAREs and regulation. Cell Mol Life Sci $65: 2814-2832$

Mancias JD, Goldberg $\mathbf{J}$ (2007) The transport signal on Sec22 for packaging into COPll-coated vesicles is a conformational epitope. Mol Cell 26:403-414

Maranda B, Brown D, Bourgoin S, Casanova JE, Vinay $P$, Ausiello DA, Marshansky V (2001) Intra-endosomal pH sensitive recruitment of the Arf-nucleotide exchange factor ARNO and Arf6 from cytoplasm to proximal tubule endosomes. J Biol Chem 276:18540-18550

Melia TJ, Weber T, McNew JA, Fisher LE, Johnston RJ, Parlati F, Mahal LK, Söllner TH, Rothman JE (2002) Regulation of membrane fusion by the membrane-proxima coil of the t-SNARE during zippering of SNAREpins. J Cell Biol 158:929-940

Meyer E, Cohen J (1999) Paramecium molecular genetics: functional complementation and homology-dependent gene inactivation. Protist 150:11-16

Momayezi M, Kersken $\mathrm{H}$, Gras U, Vilmart-Seuwen J, Plattner H (1986) Calmodulin in Paramecium tetraurelia: localization from the in vivo to the ultrastructural level. $\mathrm{J}$ Histochem Cytochem 34:1621-1638

Mossessova E, Bickford LC, Goldberg J (2003) SNARE selectivity of the COPII coat. Cell 114:483-495

Novick P, Zerial M (1997) The diversity of Rab proteins in vesicle transport. Curr Opin Cell Biol 9:496-504

Ohno S (1970) Evolution by Gene Duplication. George Allen and Unwin, London

Parlati $F$, Varlamov O, Paz K, McNew JA, Hurtado D, Söliner TH, Rothman JE (2002) Distinct SNARE complexes mediating membrane fusion in Golgi transport based on combinatorial specificity. Proc Natl Acad Sci USA 99: 5424-5429

Paumet F, Rahimian V, Rothman JE (2004) The specificity of SNARE-dependent fusion is encoded in the SNARE motif. Proc Natl Acad Sci USA 101:3376-3380

Pfeffer SR (2007) Unsolved mysteries in membrane traffic. Annu Rev Biochem 76:629-645
Plattner H (2002) My favorite cell - Paramecium. BioEssays 24:649-658

Plattner H, Hentschel J (2006) Sub-second cellular dynamics: time-resolved electron microscopy and functional correlation. Int Rev Cytol 255:133-176

Plattner H, KissmehI R (2003) Dense-core secretory vesicle docking and exocytotic membrane fusion in Paramecium cells. Biochim Biophys Acta Mol Cell Res 1641:183-193

Plattner H, Klauke N (2001) Calcium in ciliated protozoa sources, regulation, and calcium-regulated processes. Int Rev Cytol 201:115-208

Plattner H, Knoll G, Pape R (1993) Synchronization of Different Steps of the Secretory Cycle in Paramecium tetraurelia: Trichocyst Exocytosis, Exocytosis-coupled Endocytosis and Intracellular Transport. in Plattner $\mathrm{H}$ (ed) Membrane Traffic in Protozoa. JAl Press, Greenwich (CT) London, pp 123-148

Pobbati AV, Stein A, Fasshauer D (2006) N- to C-terminal SNARE complex assembly promotes rapid membrane fusion. Science 313:673-676

Quetlas S, Iborra C, Sasakawa N, De Haro L, Kumakura K, Sato K, Leveque C, Seagar M (2002) Calmodulin and lipid binding to synaptobrevin regulates calcium-dependent exocytosis. EMBO J 21:3970-3979

Rizo J, Südhof TC (2002) Snares and munc 18 in synaptic vesicle fusion. Nature Rev Neurosci 3:641-653

Rizo J, Chen X, Arac D (2006) Unraveling the mechanisms of synaptotagmin and SNARE function in neurotransmitter release. Trends Cell Biol 16:339-350

Rosenbaum JL, Witman GB (2002) Intraflagellar transport. Nature Rev Mol Cell Biol 3:813-825

Rossi V, Banfield DK, Vacca M, Dietrich LEP, Ungermann C, D'Esposito M, Galli T, Filippini F (2004) Longins and their longin domains: regulated SNAREs and multifunctional SNARE regulators. Trends Biochem Sci 29:682-688

Rothman JE (1994) Mechanisms of intracellular protein transport. Nature 372:55-63

Ruiz F, Vayssié L, Klotz C, Sperling L, Madeddu L (1998) Homology-dependent gene silencing in Paramecium. Mo Biol Cell 9:931-943

Sanderfoot A (2007) Increases in the number of SNARE genes parallels the rise of multicellularity among the green plants. Plant Physiol 144:6-17

Schilde C, Lutter K, Kissmehl R, Plattner H (2008) Molecular identification of a SNAP-25-like SNARE protein in Paramecium. Eukaryot Cell 7:1387-1402

Schilde C, Schönemann B, Sehring IM, Plattner H (2010) Distinct subcellular localization of a group of synaptobrevinlike SNAREs in Paramecium tetraurelia and effects of silencing of the SNARE-specific chaperone NSF. Eukaryot Cell 9:288-305

Schilde C, Wassmer T, Mansfeld J, Plattner H, KissmehI A (2006) A multigene family encoding R-SNAREs in the ciliate Paramecium tetraurelia. Traffic 7:440-455 
Schroeder CC, Fok AK, Allen RD (1990) Vesicle transport along microtubular ribbons and isolation of cytoplasmic dynein from Paramecium. J Cell Biol 111: 2553-2562

Schwartz JH, Li G, Suri V, Ross JJ, Alexander EA (2007) Role of SNAREs and $\mathrm{H}^{+}$-ATPase in the targeting of proton pump-coated vesicles to collecting duct cell apical membrane. Kidney Int 72:1310-1315

Skouri F, Cohen J (1997) Genetic approach to regulated exocytosis using functional complementation in Paramecium: identification of the ND7 gene required for membrane fusion. Mol Biol Cell 8:1063-1071

Soldati T, Schliwa M (2006) Powering membrane traffic in endocytosis and recycling. Nature Rev Mol Cell Biol 7: 897-908

Söllner T, Whiteheart SW, Brunner M, Erdjument-Bromage H, Geromanos S, Tempst P, Rothman JE (1993) SNAP receptors implicated in vesicle targeting and fusion. Nature 362:318-324

Sørensen JB, Wiederhold K, Müller EM, Milosevic I, Nagy G, De Groot BL, Grubmüller H, Fasshauer D (2006) Sequential $\mathrm{N}$ - to $\mathrm{C}$-terminal SNARE complex assembly drives priming and fusion of secretory vesicles. EMBO J 25:955-966

Sutton RB, Fasshauer D, Jahn R, Brunger AT (1998) Crystal structure of a SNARE complex involved in synaptic exocytosis at 2.4 A resolution. Nature 395:347-353

Thorngren N, Collins KM, Fratti RA, Wickner W, Merz AJ (2004) A soluble SNARE drives rapid docking, bypassing ATP and Sect7/18p for vacuole fusion. EMBO J 23:2765-2776

Tominaga T, Allen RD, Naitoh Y (1998) Electrophysiology of the in situ contractile vacuole complex of Paramecium reveals its membrane dynamics and electrogenic site during osmoregulatory activity. J Exp Biol 201:451-460

Ungermann C, Langosch D (2005) Functions of SNAREs in intracellular membrane fusion and lipid bilayer mixing. J Cell Sci 118:3819-3828
Vayssié L, Skouri F, Sperling L, Cohen J (2000) Molecular genetics of regulated secretion in Paramecium. Biochimie 82:269-288

Wassmer T, Sehring IM, KissmehI R, Plattner H (2009) The V-ATPase in Paramecium: functional specialization by multiple gene isoforms. Pflugers Arch Eur J Physiol 457:599-607

Weninger K, Bowen M, Choi UB, Chu S, Brunger AT (2008) Accessory proteins stabilize the acceptor complex for synaptobrevin, the 1:1 syntaxin/SNAP-25 complex. Structure 16:308-320

Whiteheart SW, Schraw T, Matveeva EA (2001) N-ethylmaleimide sensitive factor (NSF) structure and function. Int Rev Cytol 207:71-112

Wojcik SM, Brose N (2007) Regulation of membrane fusion in synaptic excitation-secretion coupling: speed and accuracy matter. Neuron $55: 11-24$

Wolfe K (2004) Evolutionary genomics: Yeasts accelerate beyond BLAST. Curr Biol 14:R392-R394

Yoshizawa AC, Kawashima S, Okuda S, Fujita M, Itoh M, Moriya Y, Hattori M, Kanehisa M (2006) Extracting sequence motifs and the phylogenetic features of SNAREdependent membrane traffic. Traffic 7:1104-1118

Zagulski M, Nowak JK, LeMouel A, Nowacki M, Migdalski A, Gromadka R, Noel B, Blanc I, Dessen P, Wincker P, Keller A-M, Cohen J, Meyer E, Sperling L (2004) High coding density on the largest Paramecium tetraurelia somatic chromosome. Curr Biol 14:1397-1404

Helmut Plattner

Department of Biology, University of Konstanz, P.O. Box 5560, 78457 Konstanz, Germany

fax +497531882245 e-mail helmut.plattner@uni-konstanz.de 